\title{
THE GLOBAL IMPACT OF HAND HYGIENE CAMPAIGNING
}

\author{
C Kilpatrick (kilpatrickc@who.int)1, B Allegranzi¹, D Pittet ${ }^{1,2}$ \\ 1. World Health Organization (WHO) Patient Safety, WHO Headquarters, Geneva, Switzerland \\ 2. Infection Control Programme, University of Geneva Hospitals and Faculty of Medicine, Geneva, Switzerland
}

Improving and sustaining hand hygiene is a long-term challenge, as those who are already involved in efforts of improvement are aware. Strategies need to be applied on many levels and include training and the change of behaviour and culture. These strategies take many years to implement and embed within healthcare settings. On 5 May, the World Health Organization (WHO) highlights the importance of hand hygiene and launches guidelines and tools on hand hygiene, based on the next phase of a patient safety work programme 'Save LIVES: Clean Your Hands'.

Since 2005, the WHO 'First Global Patient Safety Challenge' has aimed to promote and support a multimodal improvement strategy for hand hygiene, as Magiorakos et al. highlight in the opening of their paper on national hand hygiene campaigns in Europe, 20002009, published in this issue of Eurosurveillance [1].

The first phase of the 'Clean Care is Safer Care', patient safety work programme (2005-2008) saw the following initiatives under the 'First Global Patient Safety Challenge' come to fruition:

- Some 120 countries have pledged to address healthcareassociated infection through cleaner, safer care. Many of these have undertaken a range of activities since pledging;

- The 'Advanced Draft Guidelines on Hand Hygiene in Health Care', published in 2006 [2] and a suite of implementation tools have been developed and tested. This included support for eight pilot sites and over 300 additional, complementary test sites as well as a review of the current evidence and the involvement of a core group of international experts. The finalised guidelines are designed to present WHO member states and all professionals in the infection control specialty with evidence-based direction on how to improve hand hygiene compliance in the short, medium and long term. They also aim to direct on how to prevent infections and reduce the burden of clinical disease, to which poor hand hygiene contributes;

- Global awareness was raised regarding healthcare-associated infections and how the implementation of multimodal improvement strategies can contribute to their reduction;

- The creation of a global network of campaigning nations has been supported in order to share knowledge and build solidarity between those committed to improving hand hygiene in healthcare facilities.

"National programmes do not necessarily employ campaign approaches; however, national health improvement programmes have been shown in many cases to use elements of campaigning and mass media involvement to good effect" [3]. Other recent healthcare campaigns with demonstrable success, have focused not only on hand hygiene but have also included for example, prudent use of antibiotics [4].

The 'First Global Patient Safety Challenge' has, over the last three years, attempted to track the activities of national campaigns. It is encouraging to observe Magiorakos et al. additionally acknowledging the importance and value of undertaking such activities and being in communication with those in their regions who are actively working on hand hygiene improvement.

In 2007, WHO conducted its first survey and meeting of campaigning nations. Seventeen countries reported to be undertaking 20 national or sub-national campaigns [5]. In 2009, a similar survey was conducted and a total of 38 nations and sub-nations with campaigns have been recorded. Those with responsibility for leading these campaigns have been identified and information has also been gathered on whether these campaigns are 'stand alone' or part of wider healthcare associated activities and work programmes. A report of the 2009 survey will be published in the coming months.

Magiorakos et al. note that activities have taken place irrespective of whether the countries had already pledged to WHO to reduce healthcare-associated infections through cleaner safer care or not. Their article adds to the current body of knowledge on such activities. It is also important to note that $70 \%$ of campaign coordinators acknowledge the importance of the WHO pledge as a catalyst, and $89 \%$ and $73 \%$, respectively, state that the $\mathrm{WHO}$ guidelines and implementation tools for hand hygiene improvement are used as a reference (WHO, unpublished data). However, the 'First Global Patient Safety Challenge' recognised at an early stage that pledging and other publicised activities do not always lead to action at the point of care. In addition, national campaigns, once started, do not always continue.

A WHO Patient Safety 2009 initiative has been established to catalyse progress and to further move action from pledging to the point of patient care. This will be the next phase of the 'First Challenge's work on Clean Care is Safer Care' [6].

This initiative 'SAVE LIVES: Clean Your Hands' has, as of April 2009 , seen a total of 3,863 healthcare facilities registering their interest and commitment, which equates to a combined staff of over 3.6 million people. The healthcare facilities are based in different countries and territories and represent an increasing level of engagement in the global push to highlight hand hygiene as one of the best ways of reducing healthcare-associated infections. 
On 5 May, 2009:

- WHO 'Guidelines on Hand Hygiene in Health Care' will be formally launched. The guidelines feature the steps required for a national strategy for action on hand hygiene improvement;

- The revised 'Guide to Implementation' and an associated toolkit will also be launched

- The revised web pages featuring a wide range of updated information that should support all those campaigning for improved hand hygiene will go live.

Government pledging, and at times associated funding, as described for some of the countries in the article by Magiorakos et al. [1], continues to have its place. On 5 May 2009, France will become the most recent country to sign the WHO pledge.

Moving forward, WHO's 'First Global Patient Safety Challenge' aims to publish a range of scientific articles featuring data from the activities at the collaborative pilot sites in each of the WHO regions. In addition, it intends to present an overview of these data at a patient safety event in London on 15 December 2009. The 'First Challenge' team also aims to continue to promote and support the 'SAVE LIVES: Clean Your Hands' initiative on 5 May every year. The vision for this annual event is that each country and where appropriate each healthcare facility, would present and celebrate their advances in hand hygiene improvement and the impact that this had on reducing the burden of disease attributable to healthcare-associated infections. At the same time, overview and country-specific articles such as the one by Magiorakos et al., would be truly valuable and add to the evidence base of infection prevention and control.

Sustainability of hand hygiene compliance is a long way off. Working collaboratively both locally and globally will ensure that lessons can be learned and the best efforts can be made to save lives through clean hands.

\section{References}

1. Magionakos AP, Suetens C, Boyd L, Costa C, Cunney R, Drouvot V, et al. National hand hygiene campaigns in Europe 2000 - 2009. Available from: http://www. eurosurveillance.org/ViewArticle.aspx?ArticleId =19190

2. World Health Organization. Guidelines on hand hygiene in health care (advanced draft). Global patient safety challenge 2005-2006: "Clean care is safer care". Geneva: WHO; 2006. Available from: http://www.who.int/patientsafety/ events/05/HH_en.pdf

3. Perz JF, Craig AS, Coffey CS, Jorgensen DM, Mitchel E, Hall S, et al. Changes in antibiotic prescribing for children after a community-wide campaign. JAMA. 2002;287(23):3103-9.

4. Allegranzi B, Storr J, Dziekan G, Leotsakos A, Sax H, Pittet D, et al. The 1st Global Patient Safety Challenge (GPSC) catalyzing hand hygiene (HH) national campaigns worldwide. (Oral presentation) "47th Interscience Conference on Antimicrobial Agents and Chemotherapy'. Chicago; 2007 Sep 13-20.

5. WHO World Alliance for Patient Safety. Report of the First Meeting of Representatives from countries running national/sub-national hand hygiene campaigns. Geneva: WHO; 2007. Available from: http://www.who.int/ patientsafety/events/07/ps_meeting_report_geneva_29august.pdf

6. World Health Organization. Guideline on hand hygiene in health care. Geneva: WHO; 2009. Available from: http://www.who.int/gpsc/en/ 